\title{
A Novel Miniaturized Four-Ridged Horn Antenna with Enhanced Gain
}

\author{
Yunqing Cao $\mathbb{D}^{1,2}$ Menglong Wang, ${ }^{1}$ Daoyuan Sun, ${ }^{1}$ and Dan Shan $\mathbb{D}^{1,2}$ \\ ${ }^{1}$ College of Physical Science and Technology, Yangzhou University, Yangzhou 225009, China \\ ${ }^{2}$ National Laboratory of Solid State Microstructures and School of Electronic Science and Engineering and Collaborative \\ Innovation Center of Advanced Microstructures, Nanjing University, Nanjing 210093, China
}

Correspondence should be addressed to Dan Shan; shandnju@gmail.com

Received 15 April 2021; Accepted 30 July 2021; Published 9 August 2021

Academic Editor: Renato Cicchetti

Copyright (c) 2021 Yunqing Cao et al. This is an open access article distributed under the Creative Commons Attribution License, which permits unrestricted use, distribution, and reproduction in any medium, provided the original work is properly cited.

\begin{abstract}
Miniaturization of wideband antennas has attracted much attention for its wide application in modern society. This article proposes a novel broadband miniaturized four-ridged horn antenna (FRHA) with high gain operating from 2.6 to $8.4 \mathrm{GHz}$. By filling the FRHA with the epoxy-laminated glass cloth board, the side length of the aperture realizes $48 \%$ reduction comparing to a traditional ridged horn antenna. The cuboid-shaped polyethylene lens provides good impedance matching between the antenna and the air at low frequencies and decreases the aperture phase error at high frequencies, which optimizes the radiating characteristics in the whole operating band.
\end{abstract}

\section{Introduction}

Since invented in the 1950 s, the ridged horn antennas (RHAs) have been drawing attention from worldwide for its ultrawide impedance bandwidth, simple structure, and high power capacity [1-4]. Hence, the RHAs have been widely used in electromagnetic compatibility testing systems, phased array radars, biology imaging systems, and so on [5-9]. However, the RHAs are relatively large and heavy comparing to some other antenna forms, which have limited its further utilization in the future. So, it is quite necessary to develop miniaturized RHAs. On the other hand, the radiation characteristics of RHAs also need optimization. The aperture of RHAs is determined by the largest wavelength of the bandwidth, but the phase error between the center and the edge is usually too large when operating at high frequencies, which often causes distortion of radiation patterns and decline of the normal gain. In previous reports, the addition of a dielectric lens in the RHAs is an effective way to optimize the radiation performance. For example, Turk and Keskin added a small lens inward the aperture of a standard double RHA and increased the gain by approximately $5 \mathrm{~dB}$ in the range of $9-15 \mathrm{GHz}$ and reduced the pattern distortion effectively [10]. Zhang et al. proposed a broadband double-ridged horn antenna with a dielectric lens at the aperture [11]. Due to the loaded lens, the radiation pattern distortion in the high-frequency band is restrained significantly. In 2018, Diaz and Mostafavi invented a broadband quad-ridge horn antenna with a cross-polyrod lens and a prolate spheroidal lens [12]. With the combination of lenses, the directivity of the antenna is improved efficiently. Usually, the dielectric lenses are designed in complicated shapes (parabolic, trapezoid, and so on), which make it more expensive in manufacturing and integration. Therefore, simple structure, high reliability, and low cost are the key problems for wide application of dielectric lenses. Besides, in microwave applications and radar systems, it is more popular to use dual-polarization antennas with wideband range and high power capacity, for which the four-ridged horn antenna (FRHA) is a good choice. A FRHA is the combination of two pairs of ridges (perpendicular to each other) and two feed points, and each pair of ridges and the feed point are responsible for one polarization direction. 


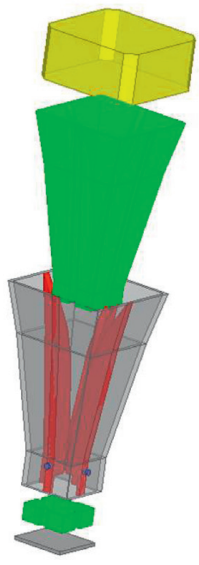

(a)

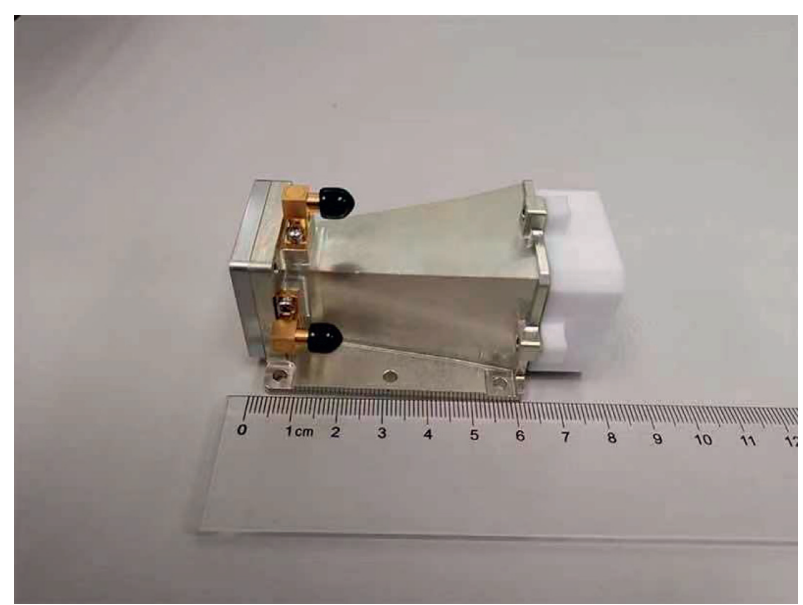

(b)

Figure 1: (a) The structure and (b) the photograph of the lens-loaded FRHA.

In this study, a novel miniaturized FRHA is presented. The FRHA is filled with the epoxy-laminated glass cloth board (ELGC, $\varepsilon_{r}=4.5$ ), and a cuboid polyethylene (PE, $\left.\varepsilon_{r}=2.2\right)$ block is added on the top as a dielectric lens. Due to the relatively high permittivity of the filling material, the side length of the aperture is reduced by $48 \%$ compared to the traditional RHAs operating at the same band, and the profile is also lowered. The simple cuboid-shaped PE lens provides perfect impedance matching between the FRHA and the air, reducing the return loss at the aperture. What is more, the dielectric lens also decreases the aperture phase error between the center and the edge, and the normal gain at high frequencies is enhanced effectively.

\section{Antenna Geometry and Design}

As shown in Figure 1(a), the miniaturized four-ridged horn antenna (FRHA) consists of the following parts: a cuboid PE block $\left(\varepsilon_{r}=2.2\right)$, the conformal ELGC filler $\left(\varepsilon_{r}=4.5\right)$ in the horn and the back cavity, a conventional metal four-ridged horn, two feeding coaxial connectors, and a metal back cover plate. The two pairs of ridges are completely identical except the location of the feeding coaxial connectors. It is worth noting that the cuboid PE block is a crucial part and acts as a lens. So, the FRHAs without or with the block are named as conventional and lens-loaded FRHAs, respectively. The photograph of the FRHA is shown in Figure 1(b). The inner edge fits the exponential equation illustrated in Figure 2(b). The detailed parameters shown in Figure 2(a) and 2(b) are listed in the explanatory text.

For a traditional ridged horn antenna, the aperture size is determined by the operating bandwidth. Usually, the side length of the aperture is half the wavelength of the lowest frequency. In this case, the operating bandwidth is from 2.6 to $8.4 \mathrm{GHz}$, which means the side length is over $58 \mathrm{~mm}$. In our case, the side length is only $30 \mathrm{~mm}$, reaching an aperture size reduction of $48 \%$. The miniaturization of the FRHA can be attributed to the filling ELGC with a relatively high permittivity. However, due to the large difference in permittivity between ELGC and air, impedance mismatching and strong reflection occurs at the interface, resulting in a high voltage standing wave ratio (VSWR). The problem will be discussed in the following.

\section{Results and Discussion}

The FRHA is designed to operate from 2.6 to $8.4 \mathrm{GHz}$. For better explanation of the dielectric lens, two kinds of FRHAs, the conventional FRHA (without the lens) and the lensloaded FRHA (with the lens), are compared. Figure 3 shows the simulated VSWR data of the conventional and lensloaded FRHAs. Besides, for better comparison, the simulated and measured data of conventional and lens-loaded FRHAs are shown in Figure 4.

We can find that the measured data fit well with the simulated data in Figure 4, and the VSWRs of the conventional FRHAs are too high for application in the operating bandwidth. As mentioned above, the large difference in permittivity between ELGC and air causes the impedance mismatching at the aperture and high return loss. Usually, the quarter-wavelength transformer is a useful and practical method for impedance matching. For the quarter-wavelength transformer, the characteristic impedance is the geometric mean of the load and source impedances. In a lossless medium, the intrinsic impedance of the dielectric can be calculated by the following equation:

$$
\eta=\eta_{0} \sqrt{\left(\frac{\mu_{r}}{\varepsilon_{r}}\right)}
$$

where $\eta_{0}$ is the intrinsic impedance of vacuum, and $\mu_{r}$ and $\varepsilon_{r}$ are the permeability and permittivity of the dielectric, respectively. In this case, the permittivity of ELGC is 4.5 , so the proper permittivity for the quarter-wavelength transformer is 2.1 .

The quarter-wavelength transformer for the FRHA is designed as a cubic PE block with the permittivity of 2.2. The thickness of the block is $18 \mathrm{~mm}$, corresponding to the frequency of about $4.2 \mathrm{GHz}$. The simulated and measured 

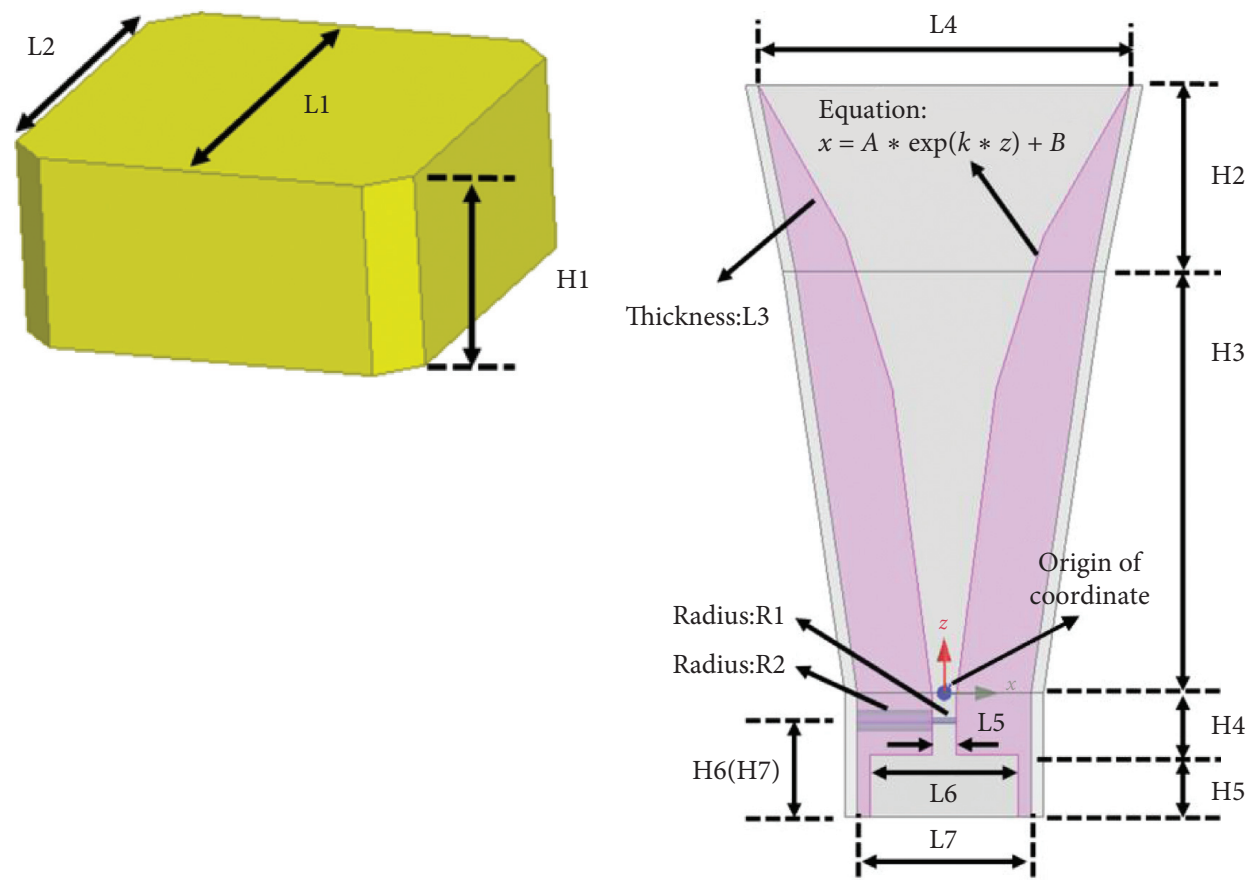

(a)

(b)

Figure 2: (a)-(b) The structure of the lens-loaded FRHA. L1 $=32 \mathrm{~mm}, \mathrm{~L} 2=26 \mathrm{~mm}, \mathrm{~L} 3=2.4 \mathrm{~mm}, \mathrm{~L} 4=30 \mathrm{~mm}, \mathrm{~L} 5=2 \mathrm{~mm}, \mathrm{~L} 6=12 \mathrm{~mm}$, $\mathrm{L} 7=14 \mathrm{~mm}, \mathrm{H} 1=18 \mathrm{~mm}, \mathrm{H} 2=15 \mathrm{~mm}, \mathrm{H} 3=34 \mathrm{~mm}, \mathrm{H} 4=5 \mathrm{~mm}, \mathrm{H} 5=5 \mathrm{~mm}, \mathrm{H} 6=7 \mathrm{~mm}, \mathrm{H} 8=7.8 \mathrm{~mm}, \mathrm{R} 1=0.25 \mathrm{~mm}, \mathrm{R} 2=0.85 \mathrm{~mm}$, $k=50 \mathrm{~m}^{-1}, A=1.322 \mathrm{~mm}$, and $B=-0.322 \mathrm{~mm}$.

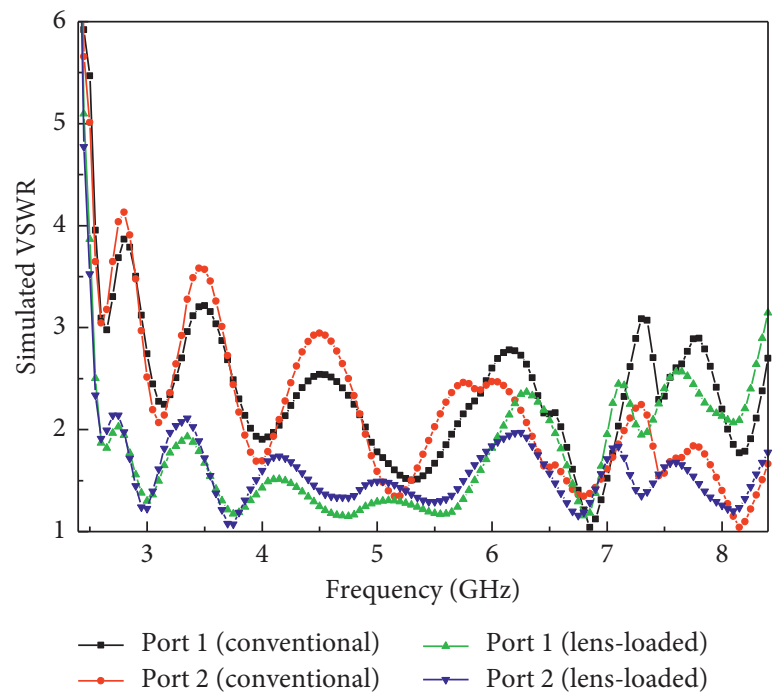

FIgURE 3: The simulated VSWR data of the conventional and lens-loaded FRHAs.

VSWR data for the lens-loaded FRHAs are shown in Figures 3 and 4. Comparing with the conventional FRHA, the VSWRs of the lens-loaded FRHA are decreased, especially in the range of $2.6-6 \mathrm{GHz}$. In the whole operating band, the measured VSWRs of the two ports are less than 2.5 except for several points. However, the VSWRs of the conventional and lens-loaded FRHAs are comparative in the high-frequency range. Usually, the quarter-wavelength transformer can only operate in a small-frequency range. In this case, the impedance matching performance can be obviously influenced by changing the thickness of the dielectric lens (not shown here). Considering that the VSWR for the conventional FRHA is worse at the low-frequency band and the size of the dielectric lens cannot be too large, the thickness is chosen to be $18 \mathrm{~mm}$.

The measured radiation patterns of conventional and lens-loaded FRHAs are shown in Figure 5. Since the radiation patterns of the two polarizations are quite consistent, 

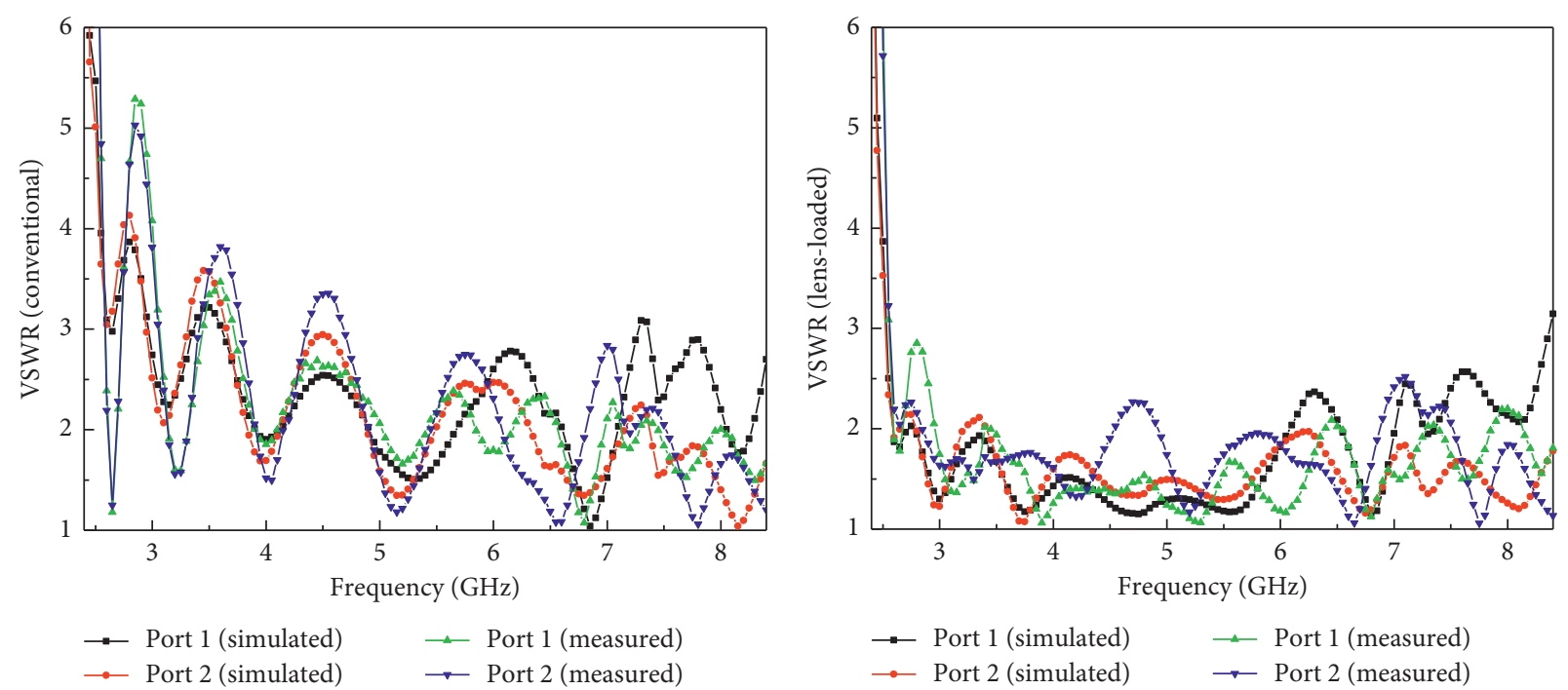

(b)

Figure 4: The simulated and measured VSWR data of (a) conventional and (b) lens-loaded FRHAs.
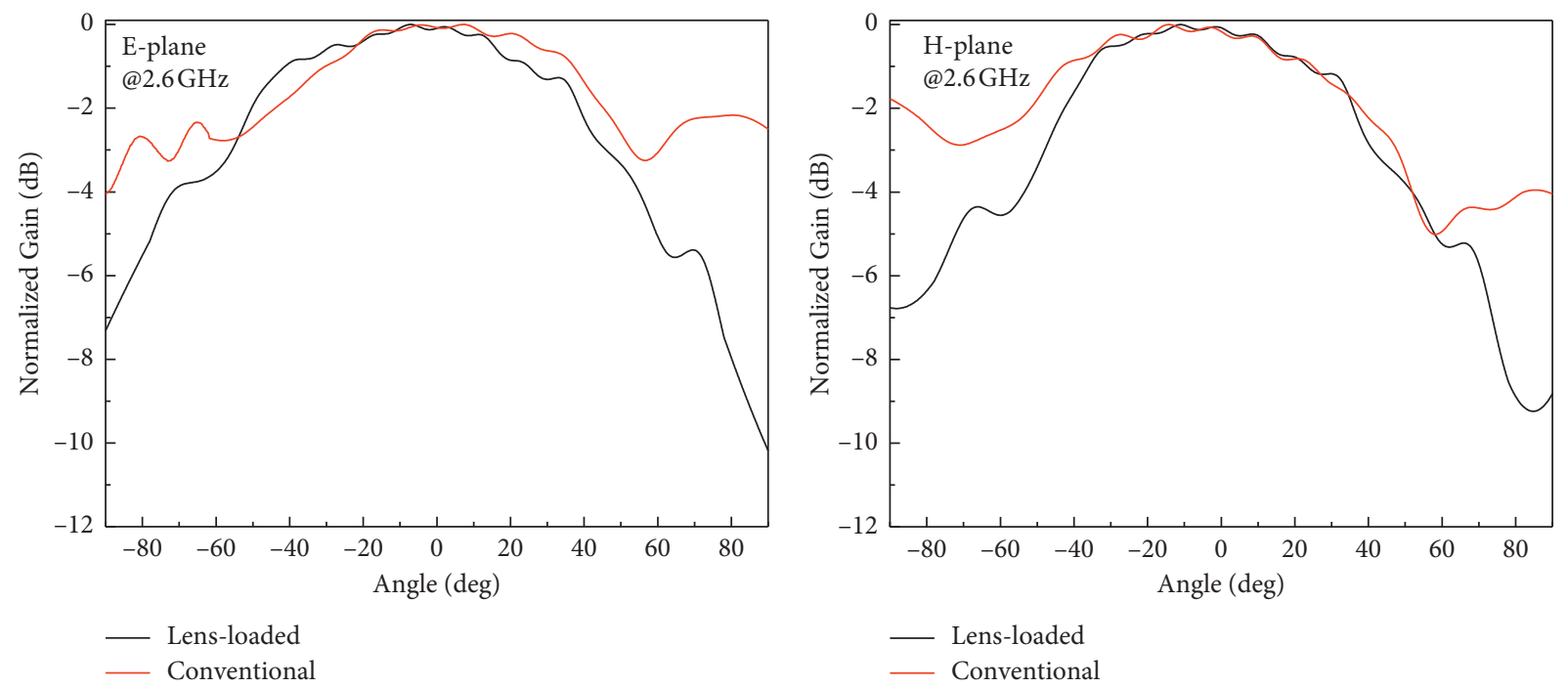

(a)

(b)

FIGURE 5: Continued. 


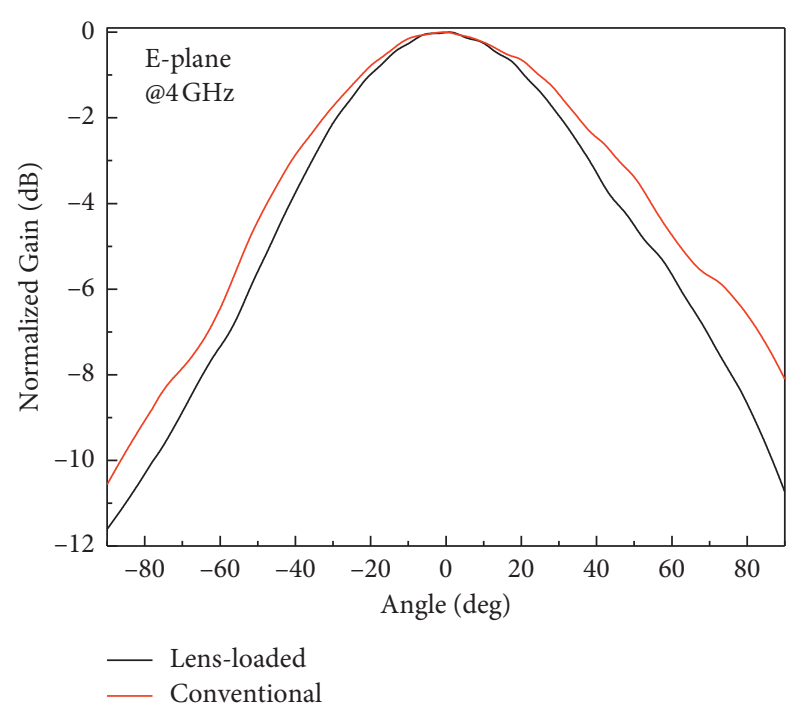

(c)

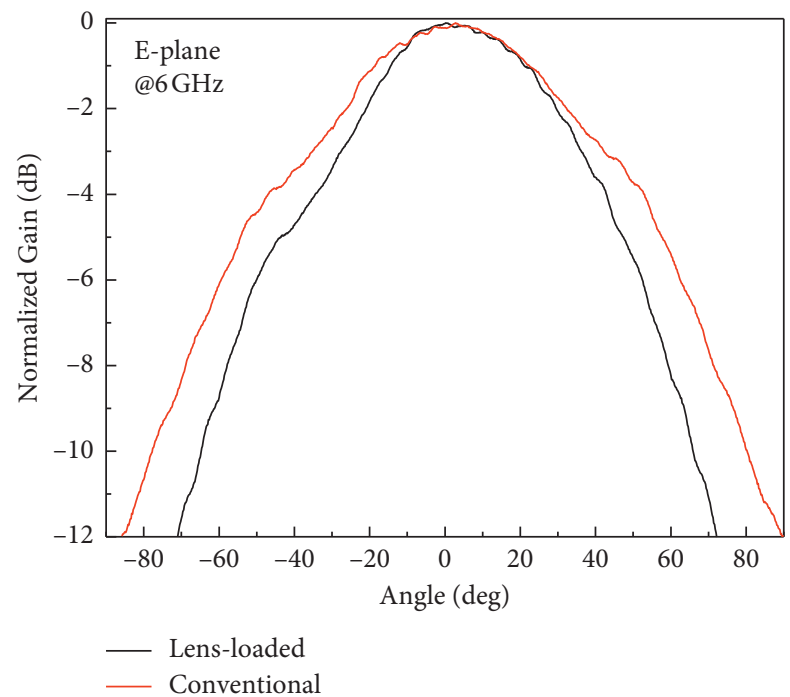

(e)

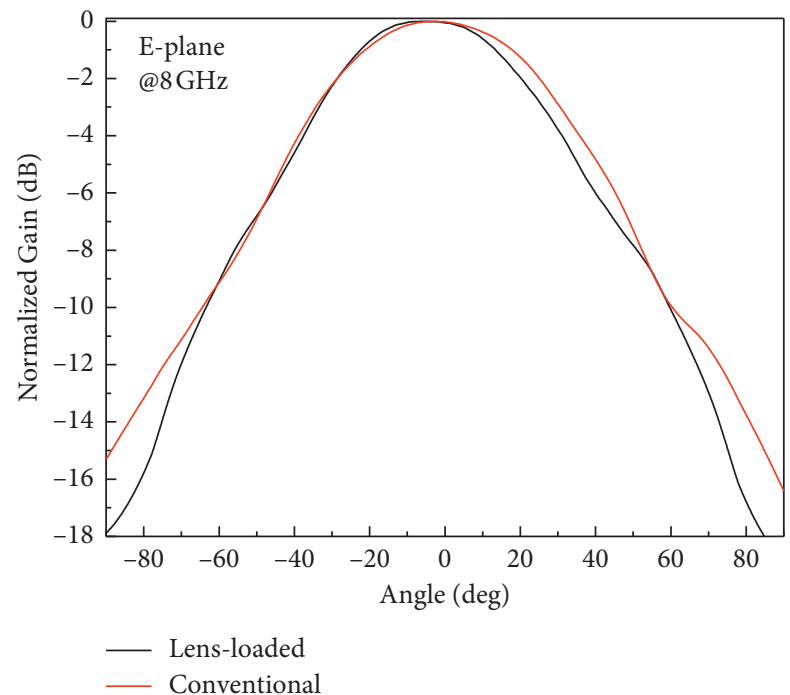

$(\mathrm{g})$

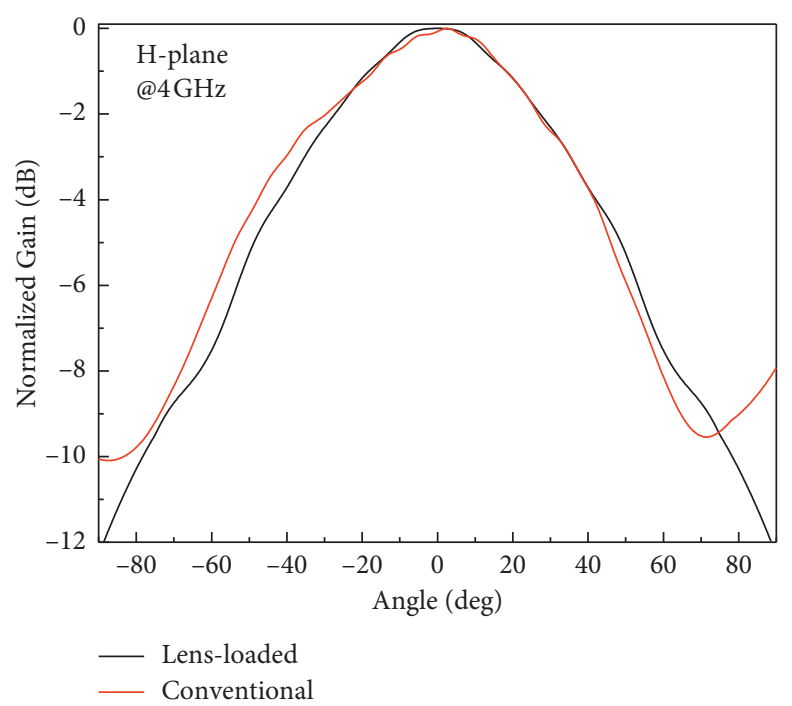

(d)

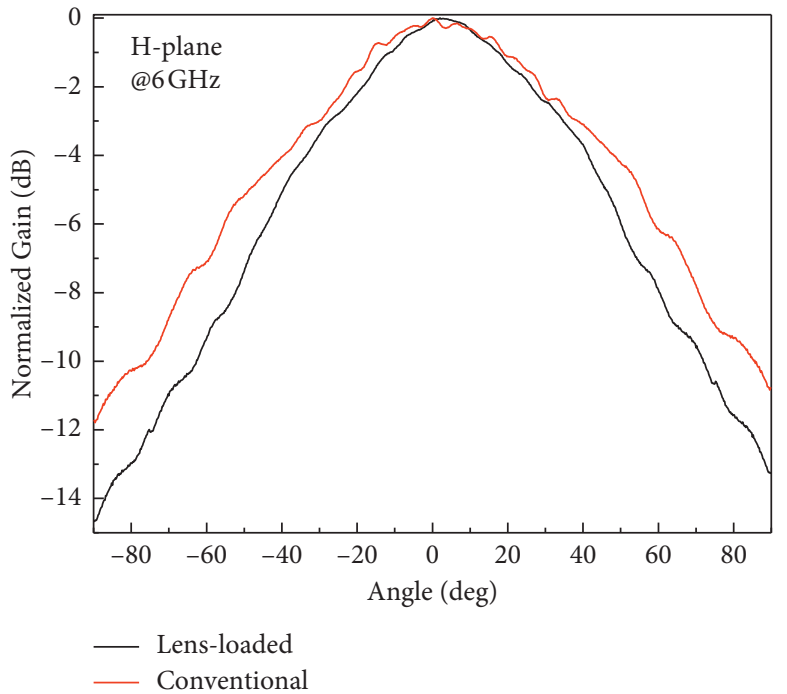

(f)

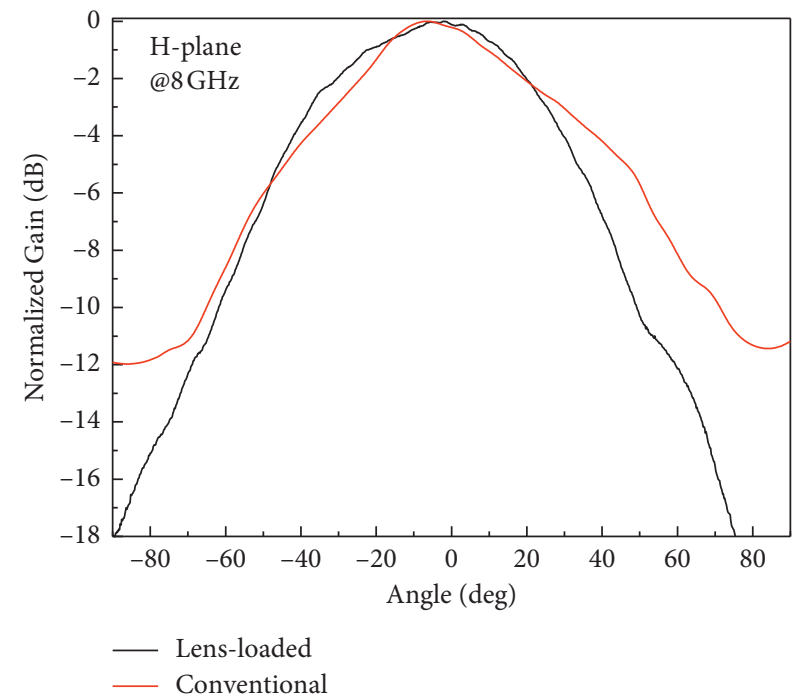

(h)

FiguRe 5: Measured radiation patterns of (a), (c), (e), (g) conventional and (b), (d), (f), (h) lens-loaded FRHAs at 2.6-8 GHz. 


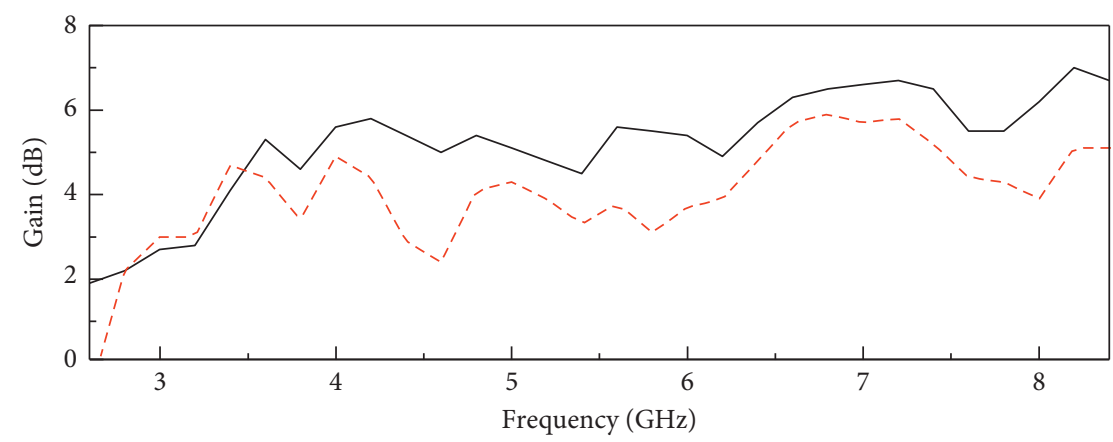

- Port 1 (lens-loaded)

- - Port 1 (conventional)

(a)

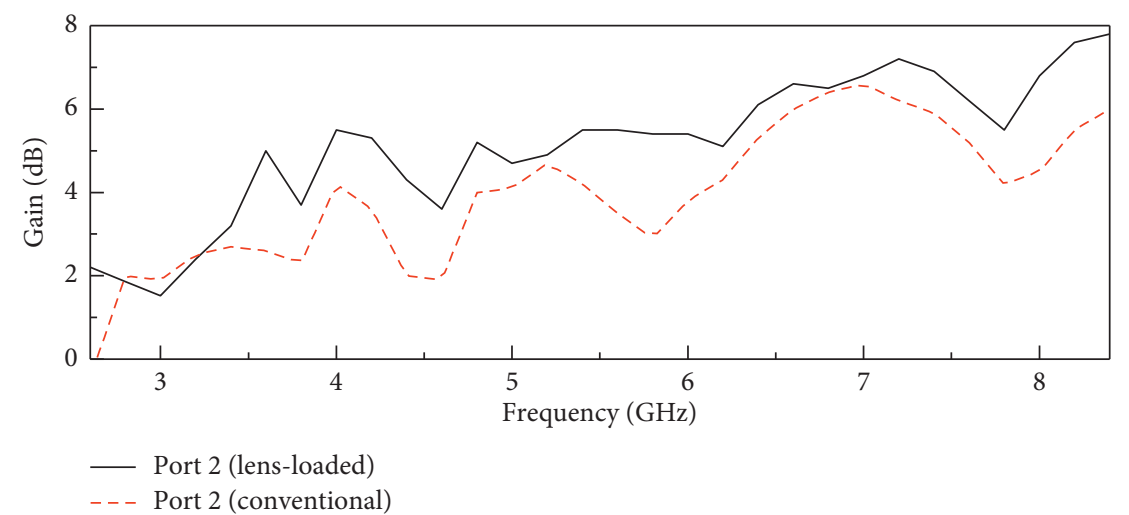

(b)

FiguRE 6: The measured normal gain data of (a) the conventional and (b) lens-loaded FRHAs.

only the radiation patterns of port 1 are shown for comparison. At $2.6 \mathrm{GHz}$, the radiation patterns of conventional FRHA are distorted and asymmetrical in both E-plane and $\mathrm{H}$-plane. Due to the high return loss, the radiating characteristics are poor, and the radiation pattern can be seriously affected by error of measurements. For the frequency of $4-8 \mathrm{GHz}$, the radiation patterns of conventional FRHA show apparently larger $3 \mathrm{~dB}$ beam width, which means lower gain than that of lens-loaded FRHAs. The dielectric lens plays another important role to improve the gain of the FRHA besides the quarter-wavelength transformer.

To better understand the performance of the dielectric lens, the normal gains are shown in Figure 6. In this figure, we can find that the lens-loaded FRHAs show better radiating characteristics than the conventional ones for both polarizations. Considering the comparatively worse VSWR of the conventional FRHAs, it is easy to understand that the gains are lower than the lens-loaded ones in the low-frequency range. However, it is little confusing that the lensloaded FRHAs also show higher gain in the high-frequency range since the VSWRs for the two kinds of antennas are comparative. For broadband ridged horn antennas, the radiation patterns at high frequencies can be influenced by the aperture size. On the one hand, the side length of the aperture is approximately half the wavelength of the low-end frequency, so a large aperture size is necessary for a

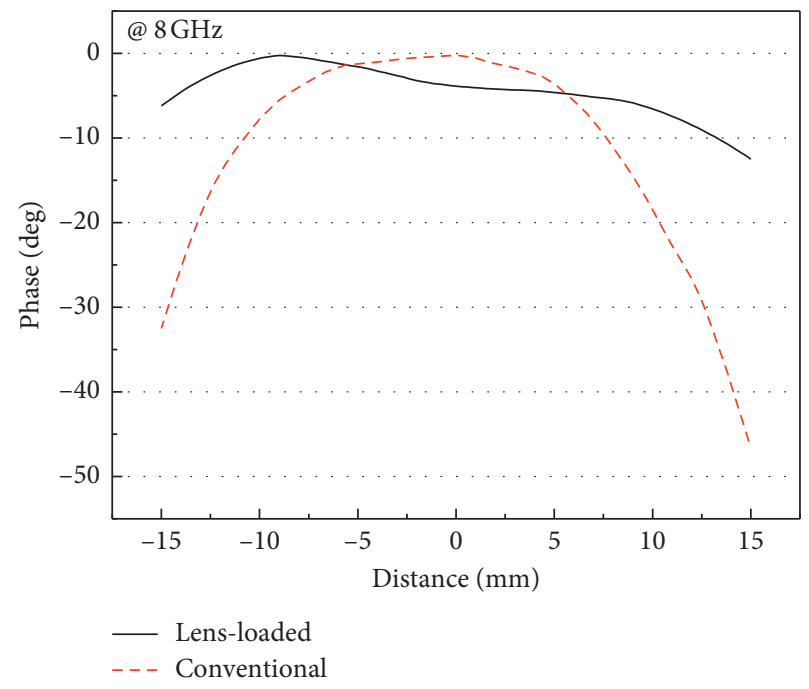

FIgURE 7: The simulated aperture phase error at $8 \mathrm{GHz}$ of the conventional and lens-loaded FRHAs.

broadband horn antenna. On the other hand, the large aperture size will lead to the generation of high-order modes and high phase error at the aperture, which will strongly deteriorate the radiating characteristics at high frequencies. In recent reports, the addition of dielectric lens is an effective 
method to optimize the radiation pattern. The dielectric lenses are often designed in specific shapes, but it makes it more expensive and more difficult to integrate with the antennas. In our studies, the dielectric lens is designed as a simple cubic block. Besides providing better impedance matching as a quarter-wavelength transformer at low frequencies, the dielectric lens also decreases the aperture phase error, especially at high frequencies. The simulated aperture phase error at $8 \mathrm{GHz}$ for the conventional and lens-loaded FRHAs is shown in Figure 7. For the conventional FRHA, the phase changes rapidly when moving away from the center, and the phase error is over $45^{\circ}$ between the center and edge of the aperture. For the lens-loaded antenna, the phase error is less than $15^{\circ}$, which will effectively enhance the aperture efficiency. Thus, the dielectric lens optimizes the radiating characteristics and enhances the gain in the entire operating band.

\section{Conclusions}

In this study, a novel broadband miniaturized FRHA operating from 2.6 to $8.4 \mathrm{GHz}$ is proposed. By filling the FRHA with ELGC, the side length of the aperture is reduced by $48 \%$ comparing to the conventional ones. Meanwhile, a simplestructured cubic PE dielectric lens is developed. The dielectric lens plays the role of a quarter-wavelength transformer at low frequencies to make better impedance matching at the aperture, and it also decreases the aperture phase error at high frequencies. Hence, the radiating characteristics of the two ports are optimized in the whole operating band.

\section{Data Availability}

The data used to support the findings of this study are available from the corresponding authors upon request.

\section{Conflicts of Interest}

The authors declare that they have no conflicts of interest.

\section{Acknowledgments}

This work was supported by the National Natural Science Foundation of China (NSFC) (61704148) and Postdoctoral Research Grant Program of Jiangsu Province (2020Z403).

\section{References}

[1] B. Christian, P. Leuchtmann, and R. Vahldieck, "Analysis and simulation of a $1-18 \mathrm{GHz}$ broadband double-ridged horn antenna," IEEE Transactions on Electromagnetic Compatibility, vol. 45, no. 1, pp. 55-60, 2003.

[2] J.-I. Moon and J.-H. Yun, "Double-ridged horn antenna for millimeter-wave applications," Microwave and Optical Technology Letters, vol. 48, no. 11, pp. 2165-2167, 2006.

[3] A. R. Mallahzadeh, A. A. Hassani, and R. H. Hamid, "A novel dual-polarized double-ridged horn antenna for wideband applications," Progress In Electromagnetics Research B, vol. 1, pp. 67-80, 2008.
[4] Z. Yue, Y. Liu, and S. Gong, "A novel 2-18 GHz double-ridged horn antenna with simple structure," Microwave and Optical Technology Letters, vol. 60, no. 7, pp. 1787-1794, 2018.

[5] M. Ghorbani and A. Khaleghi, "Double ridged horn antenna designs for wideband application," in Proceedings of the 19th Iranian Conference on Electrical Engineering, pp. 1-4, IEEE, Tehran, Iran, May 2011.

[6] B. Jacobs, J. W. Odendaal, and J. Joubert, "An improved design for a $1-18 \mathrm{GHz}$ double-ridged guide horn antenna," IEEE Transactions on Antennas and Propagation, vol. 60, no. 9, pp. 4110-4118, 2012.

[7] R. J. Fontana, "Recent system applications of short-pulse ultra-wideband (UWB) technology," IEEE Transactions on Microwave Theory and Techniques, vol. 52, no. 9, pp. 20872104, 2004.

[8] S. Latif, P. Stephen, and S. Lotfollah, "A double-ridged horn antenna design in canola oil for medical imaging," in Proceedings of the 2nd International Conference on Advances in Electrical Engineering (ICAEE), December 2013.

[9] S. Siamak, Y. Alfadhl, and X. D. Chen, "Compact ultrawideband double-ridged horn antennas for medical imaging," in Proceedings of the 2016 Loughborough Antennas \& Propagation Conference (LAPC), IEEE, Loughborough, UK, November 2016.

[10] A. S. Turk and A. K. Keskin, "Partially dielectric-loaded ridged horn antenna design for ultrawideband gain and radiation performance enhancement," IEEE Antennas and Wireless Propagation Letters, vol. 11, pp. 921-924, 2012.

[11] N. Zhang, J. H. Qiu, and P. Y. Zhang, "Design of a novel broadband EMC double ridged guide horn antenna," Progress in Electromagnetics Research, vol. 39, pp. 225-236, 2013.

[12] R. E. Diaz and M. Mostafavi, "Broadband quad-ridge horn antennas," U.S. Patent Application, 15/849, 2018. 